\title{
ARTICLE Chemogenetic modulation of accumbens direct or indirect pathways bidirectionally alters reinstatement of heroin-seeking in high- but not low-risk rats
}

\author{
Timothy J. O'Neal ${ }^{1,2,3}$, Marlaena N. Nooney ${ }^{3}$, Katie Thien ${ }^{3}$ and Susan M. Ferguson ${ }^{1,2,3,4}$
}

Opioid addiction has been declared a public health emergency, with fatal overdoses following relapse reaching epidemic proportions and disease-associated costs continuing to escalate. Relapse is often triggered by re-exposure to drug-associated cues, and though the neural substrates responsible for relapse in vulnerable individuals remains ambiguous, the nucleus accumbens (NAc) has been shown to play a central role. NAc direct and indirect pathway medium spiny neurons (dMSNs and iMSNs) can have oppositional control over reward-seeking and associative learning and are critically involved in reinstatement of psychostimulantseeking. However, whether these pathways similarly regulate reinstatement of opioid-seeking remains unknown, as is their role in modulating motivation to take opioids. Here, we describe a method for classifying addiction severity in outbred rats following intermittent-access heroin self-administration that identifies subgroups as addiction-vulnerable (high-risk) or addiction-resistant (low-risk). Using dual viral-mediated gene transfer of DREADDs, we show that transient inactivation of dMSNs or activation of iMSNs is capable of suppressing cue-induced reinstatement of heroin-seeking in high- but not low-risk rats. Surprisingly, however, the motivation to self-administer heroin was unchanged, indicating a divergence in the encoding of heroin-taking and heroin-seeking in rats. We further show that transient activation of dMSNs or inactivation of iMSNs exacerbates cue-induced reinstatement of heroin-seeking in high- but not low-risk rats, again with no effect on motivation. These findings demonstrate a critical role for dMSNs and iMSNs in encoding vulnerability to reinstatement of heroin-seeking and provide insight into the specific neurobiological changes that occur in vulnerable groups following heroin self-administration.

Neuropsychopharmacology (2020) 45:1251-1262; https://doi.org/10.1038/s41386-019-0571-9

\section{INTRODUCTION}

Drug addiction is characterized by cycles of compulsive drugtaking and drug-seeking and high rates of relapse. Relapse rates for opioids are higher than any other drug class, with up to $59 \%$ of individuals relapsing in the first week of abstinence and $80 \%$ relapsing in the first month [1]. Moreover, deaths linked to opioid overdoses are currently the leading cause of accidental death among American adults.

The cortico-basal ganglia (C-BG) circuit has been identified as a critical neural substrate in regulating the development and persistence of addiction. The striatum, which can be anatomically divided into the dorsal striatum and the nucleus accumbens (NAc), serves as the interface of the C-BG circuit, integrating cortical and subcortical inputs to guide a diverse set of behaviors including associative learning, decision-making, and motivation $[2,3]$. The NAc is necessary for the development and expression of opioidseeking $[4,5]$, and has been shown to modulate opioid reward and opioid-taking [6-9]. However, the striatum is a heterogeneous structure containing two interspersed populations of GABAergic medium spiny neurons (MSNs): direct pathway MSNs (dMSNs) and indirect pathway MSNs (iMSNs). Both dMSNs and iMSNs receive extensive glutamatergic inputs from cortical, subcortical, and thalamic nuclei; GABAergic inputs from local striatal interneurons and other MSNs via lateral inhibition; and dopaminergic inputs from the ventral tegmental area (VTA) and substantia nigra [10]. However, NAc dMSNs project directly to the VTA, while NAc iMSNs project to the ventral pallidum (VP). As a result, these neuron populations can have opposing control over behavioral output, with stimulation of dMSNs facilitating behavior by serving as a "go" signal and stimulation of iMSNs suppressing behavior by serving as a "stop" signal $[3,11]$. With respect to addiction, it has been hypothesized that disruptions in the balance of signaling between dMSNs and iMSNs (i.e., excessive "go" signal or insufficient "stop" signal) could underlie the development of the compulsive drug-taking and drug-seeking behaviors that can develop after drug use. Nonetheless, the role of these striatal cell populations has not been examined in behaviors related to opioid addiction.

As only a subset of drug users transition from casual use to addiction, it is essential to investigate whether addiction vulnerability is an intrinsic (e.g., genetically-defined) or acquired trait [12]. Thus, we have drawn from previous work investigating individual variability in addiction-like behavior [13-15] and developed a model for characterizing addiction severity using

\footnotetext{
${ }^{1}$ Graduate Program in Neuroscience, University of Washington, Seattle, WA 98195, USA; ${ }^{2}$ Department of Psychiatry \& Behavioral Sciences, University of Washington, Seattle, WA

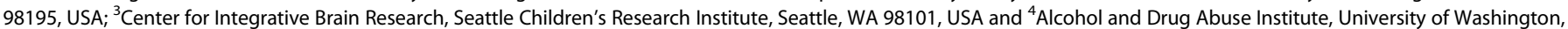
Seattle, WA 98195, USA

Correspondence: Susan M. Ferguson (smfergus@uw.edu)
}

Received: 12 July 2019 Revised: 5 November 2019 Accepted: 11 November 2019

Published online: 20 November 2019 
an intermittent-access (IntA) self-administration (SA) procedure that has been shown to produce distinct patterns of behavior in subsets of rats [14, 15]. Additionally, we incorporated key components of the DSM diagnostic criteria for Opioid Use Disorder, including difficulty stopping or limiting drug intake, high motivation to obtain and consume drug, sustained drug craving during periods of abstinence, and relapse following abstinence into our severity classification model [16]. We then combined this model with dual-viral-mediated gene transfer techniques for selective expression of Gi/o- and Gq-DREADDs in NAc dMSNs and iMSNs to determine how transient, bidirectional changes in the activity of these cell populations $[17,18]$ alters the motivation to take heroin and cue-induced reinstatement of heroin-seeking in rats expressing low and high levels of addictionlike behavior.

\section{METHODS}

Subjects

Outbred male Sprague-Dawley rats ( $n=133$; Envigo) weighing 250-274 g upon arrival were pair-housed in a temperature- and humidity-controlled vivarium on a $12 \mathrm{~h} \mathrm{light/dark} \mathrm{cycle.} \mathrm{Rats} \mathrm{were}$ mildly food-restricted ( 95-100\% of initial body weight) 2 days prior to self-administration for the study duration. Behavioral procedures occurred at the beginning of the dark cycle, and all procedures were performed according to National Institutes of Health (NIH) guidelines and approved by Seattle Children's Research Institute's Institutional Animal Care and Use Committee (IACUC).

\section{Drugs}

Diamorphine $\mathrm{HCl}$ (heroin) was obtained through the Drug Supply Program of the National Institute on Drug Abuse (NIDA) and dissolved in sterile saline $(0.9 \% \mathrm{NaCl})$ to a stock concentration of 5 $\mathrm{mg} / \mathrm{ml}$. Syringes were prepared based on body weight for administration of $0.075 \mathrm{mg} / \mathrm{kg}$ heroin per infusion $(50 \mu \mathrm{l} /$ infusion delivered over $2.8 \mathrm{~s}$ ). D-amphetamine (Millipore-Sigma; A5880) was dissolved in sterile saline at a concentration of $2 \mathrm{mg} / \mathrm{ml}$ and administered at a dose of $1 \mathrm{ml} / \mathrm{kg}$. Clozapine- $N$-oxide (CNO) was obtained through the Rapid Access to Investigate Drug Program of the National Institute of Neurological Disorders and Stroke (NINDS) of the NIH and was dissolved in dimethyl sulfoxide (DMSO; Sigma Aldrich, D650) in a hot water bath, then further diluted in sterile water to a final concentration of $6 \%$ DMSO. CNO was prepared fresh daily at a concentration of $5 \mathrm{mg} / \mathrm{ml}$ and administered ip at a dose of $1 \mathrm{ml} / \mathrm{kg}$. Vehicle (VEH) injections consisted of DMSO dissolved in sterile water to a final concentration of $6 \%$ DMSO and were administered ip at a volume of $1 \mathrm{ml} / \mathrm{kg}$.

Viral vectors

Adeno-associated viruses containing Cre-dependent Gi-coupled (pAAV-hSyn-DIO-hM ${ }_{4} \mathrm{D}(\mathrm{Gi})$-mCherry) and Gq-coupled (pAAV-hSynDIO-hM3D(Gq)-mCherry) DREADDs were acquired from Addgene (viral prep \#44362-AAV8 and \#44361-AAV8, respectively) and had titers of $\geq 4 \times 10^{12}$ viral genomes $/ \mathrm{ml}$. Adeno-associated virus containing Cre-dependent eGFP (rAAV5-hSyn-DIO-eGFP) was acquired from the UNC Vector Core (lot AV4497c) and had a titer of $4.1 \times 10^{21}$ viral molecules $/ \mathrm{ml}$. Canine adenovirus containing retrogradely-transported Cre (CAV2-Cre) had a titer of $\sim 2.5 \times 10^{9}$ viral genomes $/ \mu \mathrm{l}$ and was prepared as previously described [19].

\section{Surgical procedures}

Rats were anesthetized with isoflurane $(1-5 \%$ inhaled; Patterson Veterinary) and received meloxicam $(1 \mathrm{mg} / \mathrm{ml}, 1 \mathrm{ml} / \mathrm{kg}$ subcutaneously; Patterson Veterinary) for analgesia. Rats were monitored for at least 3 days following each surgical procedure.
Catheter surgeries. Jugular catheterization surgeries were performed as previously described [20]. Catheters were flushed daily with gentamicin $(5 \mathrm{mg} / \mathrm{ml})$ and heparin $(3 \mathrm{U} / \mathrm{ml})$ in sterile saline $(0.2 \mathrm{ml} /$ day, iv) to maintain patency. Catheter patency was checked prior to the first day of SA and following the final PR test with brevital sodium $(10 \mathrm{mg} / \mathrm{ml}$ in sterile saline, $0.05-0.2 \mathrm{ml} /$ infusion, iv; methohexital sodium, Patterson Veterinary); rats that became ataxic within $5 \mathrm{~s}$ of infusion were considered to have maintained patency. Rats that lost patency $(n=19)$ or whose catheters were damaged $(n=6)$ prior to the beginning of extinction training were excluded from all analyses.

Stereotaxic surgeries. Stereotaxic surgeries were performed using standard procedures [20]. Viral vectors were delivered via 33gauge needles and infused at a volume of $500 \mathrm{nl} / \mathrm{virus}$ and a rate of $100 \mathrm{nl} / \mathrm{min}$, unless otherwise specified. Coordinates for target brain regions (in $\mathrm{mm}$, relative to bregma) were as follows: NAc $[\mathrm{A} / \mathrm{P}+1.6, \mathrm{M} / \mathrm{L} \pm 1.5, \mathrm{D} / \mathrm{V}-7.3], \mathrm{VTA}[\mathrm{A} / \mathrm{P}-5.0, \mathrm{M} / \mathrm{L} \pm 0.9, \mathrm{D} / \mathrm{V}-8.5]$, VP $[A / P-0.1, M / L \pm 2.0, D / V-8.0]$ [21]. For c-Fos experiments, CAV2-Cre was bilaterally infused into the VTA or VP, DIO-hM4Di or DIO-hM3Dq was unilaterally infused into the left NAC, and DIOeGFP was unilaterally infused into the right NAc. For experiments targeting dMSNs, CAV2-Cre was bilaterally infused into the VTA and DIO-hM4Di or DIO-hM3Dq was bilaterally infused into the NAc. For experiments targeting iMSNs, CAV2-Cre was bilaterally infused into the VP and DIO-hM4Di or DIO-hM3Dq was bilaterally infused into the NAc.

\section{Self-administration and severity classification}

Self-administration apparatus. Training and testing were conducted in self-administration chambers equipped with two retractable levers, white cue lights located above each lever, a house light, and a grid floor (MedAssociates). A syringe pump located outside each chamber was connected to a swivel within each chamber to permit iv drug infusions.

Self-administration procedure. Rats were trained to selfadminister heroin under an IntA schedule that produces spikes in brain drug concentration [14]. Each SA session (6 h/day, 10 days) consisted of 12 repeating drug-available $(5 \mathrm{~min})$ and drugunavailable $(25 \mathrm{~min})$ blocks; the length of each drug-unavailable block ensured that brain concentrations for heroin and its two active metabolites (morphine and 6-monoacetylmorphine) were on the descending limb of their pharmacokinetic curves at the beginning of each drug-available block [22]. Daily IntA SA sessions were signaled by extinction of house lights and extension of two levers into the chamber: lever presses on the active lever resulted in drug delivery (FR1, $0.075 \mathrm{mg} / \mathrm{kg} /$ infusion over $2.8 \mathrm{~s}$ ) and illumination of the cue light $(3 \mathrm{~s})$, while lever presses on the inactive lever had no consequences. At the end of each drugavailable block, house lights re-illuminated to signal the beginning of the drug-unavailable block and levers remained extended to monitor drug-seeking during cued-unavailability, though lever presses resulted in neither drug delivery nor cue presentation. Following IntA SA, rats underwent a progressive ratio (PR) test in which the number of lever presses required for an infusion increased with each successive infusion $(5,10,20,45,65,85,115$, $145,165,185,215,245)$ until either $30 \mathrm{~min}$ passed without an infusion or $6 \mathrm{~h}$ total had elapsed [20]. Next, rats underwent daily extinction sessions ( $60 \mathrm{~min} /$ day, 9 days) during which lever presses no longer resulted in cue presentation or drug delivery; this was sufficient to reduce the responding of all rats to $<20 \%$ of their final day of SA. Following extinction training, rats underwent a cueinduced reinstatement test $(60 \mathrm{~min})$, during which the drug-paired cue light was presented at the beginning of the session and active lever presses resulted in further cue presentations but no drug delivery. 
Addiction severity classification. Six behavioral measures were collected and used to classify rats according to their overall addiction severity: (1) Intake, calculated as the total number of heroin infusions taken during IntA SA; (2) Consistency, calculated as the cumulative percentage of drug-available blocks across all IntA sessions in which at least one infusion was self-administered; (3) Seeking, calculated as the cumulative number of active presses during drug-unavailable blocks across all IntA sessions; (4) Motivation, calculated as the breakpoint during PR testing; (5) Extinction, calculated as the total number of active lever presses during extinction training; and (6) Relapse, calculated as the total number of active lever presses during cue-induced reinstatement. The raw values for each behavior were converted to z-scores by subtracting the group mean from the individual values and dividing by the standard deviation of the group [23]. Individual zscores for each animal were combined to give a cumulative addiction severity score (SS), which was used to classify rats into low-risk (SS $<-1)$, moderate-risk $(-1 \leq \mathrm{SS} \leq 1)$, and high-risk $(\mathrm{SS}>1)$. The goal of this study was to examine neurobiological differences between addiction-vulnerable (high-risk) and addiction-resilient (low-risk) rats, so moderate-risk rats $(n=11)$ were excluded.

\section{Anatomical tracing}

To validate that DREADDs were selectively targeted to dMSNs, cholera toxin subunit B (CTB) conjugated to AlexaFluor (AF) 647 (CTB-647; C-34778, ThermoFisher) was mixed 1:1 with CAV2-Cre and infused into the VTA (700 $\mathrm{nl}$ total, $70 \mathrm{nl} / \mathrm{min})$, CTB conjugated to AF488 (CTB-488; C-34775, ThermoFisher) was infused into the VP (350 $\mathrm{nl}, 70 \mathrm{nl} / \mathrm{min})$, and DIO-hM4Di-mCherry was infused into the NAc. To validate that DREADDs were selectively targeted to iMSNs, CTB-647 was mixed 1:1 with CAV2-Cre and co-infused into the VP (700 nl total, $70 \mathrm{nl} / \mathrm{min}), \mathrm{CTB}-488$ was infused into the VTA $(350 \mathrm{nl}$ total, $70 \mathrm{nl} / \mathrm{min})$, and DIO-hM4Di-mCherry was infused into the NAc. Twenty-one days later, rats were anesthetized with Beuthanasia-D ( $2 \mathrm{ml} / \mathrm{kg}$ ip; Patterson Veterinary) and transcardially perfused with phosphate-buffered saline (PBS; $\mathrm{pH}=7.4$ ) followed by paraformaldehyde (PFA; $4 \%$ in PBS). Brains were extracted, fixed overnight in PFA, post-fixed for $>48 \mathrm{~h}$ in sucrose $(30 \%$ in PBS), and sectioned $(50 \mu \mathrm{m})$ with a vibrating microtome. Z-stacks along the rostral/caudal axis of the NAc $(\mathrm{A} / \mathrm{P}+2.5$ through $\mathrm{A} / \mathrm{P}$ $+0.7)$ were collected with confocal microscopy $(\times 20$; Zeiss LSM 710), and neuronal localization of CTB-488, CTB-647, and hM4DimCherry was quantified using ImageJ (V1.49; NIH) and Adobe Illustrator (CC 2019).

Chemogenetic modulation of amphetamine-stimulated c-Fos To confirm that activation of Gi/o- and Gq-coupled DREADDs modulates activity of NAc dMSNs and iMSNs, c-Fos was measured following DREADD activation in an amphetamine + novelty task that has previously been shown to induce c-Fos in both dMSNs and iMSNs [24]. Rats were injected with CNO (5 mg/kg, ip) and returned to their home cages. Thirty min later, rats were injected with d-amphetamine ( $2 \mathrm{mg} / \mathrm{kg}$, ip), and placed into novel operant boxes. Fifty-five min later, rats were anesthetized and perfused, and brain sections were collected as described above. To visualize c-Fos, floating sections were washed (PBS; $3 \times 10 \mathrm{~min}$ ), blocked $(0.25 \%$ Triton-X, 5\% normal goat serum, PBS; $120 \mathrm{~min})$, and incubated with a primary antibody (1:800 rabbit anti-c-Fos, $0.25 \%$ Triton-X, 2.5\% normal goat serum, PBS; 24 h; Cell Signaling \#2250S, RRID: AB_2247211). Sections were then washed (PBS; $3 \times 10 \mathrm{~min}$ ) and incubated with a secondary antibody conjugated to AF-647 (1:500 goat-anti-rabbit, $0.25 \%$ Triton- $X, 2.5 \%$ normal goat serum, PBS; 120 min; Life Technologies \#A32733, RRID: AB_2633282). Finally, sections were washed (PBS; $3 \times 10 \mathrm{~min}$ ), mounted on slides, and coverslipped with Vectashield mounting medium with DAPI (Vector Labs). Tri-color z-stacks (20x magnification) along the rostral/caudal axis of the NAc $(A / P+2.7$ through $A / P+0.5)$ were collected via confocal microscopy and c-Fos + cells were counted by three researchers who were blinded to experimental conditions.

Chemogenetic modulation of addiction-like behaviors

To assess the role of NAc dMSNs and iMSNs in driving motivation and relapse in low- and high-risk rats, excitatory (Gq-coupled; hM3Dq) or inhibitory (Gi/o-coupled; hM4Di) DREADDs were transiently activated with CNO prior to PR and cue-induced reinstatement tests. Gq-DREADDs were used because they directly increase neuronal firing via calcium channel activation, rather than the more broad effects that Gs-DREADDs have on neuronal activity through changes in gene transcription, protein trafficking, etc [17]. In addition, Gs-DREADDs can have some constitutive activity $[25,26]$. CNO and VEH injections were administered $30 \mathrm{~min}$ prior to test sessions in a pseudo-randomized, within-subject design. Additional days of SA (3) and extinction (6) were interleaved between PR and cue-induced reinstatement tests, respectively, to re-baseline behavior. Unpaired $t$-tests (VEH first vs VEH second) revealed no significant effect of session order on progressive ratio $\left(t_{(75)}=1.56, p=0.12\right)$ or cue-induced reinstatement of drug-seeking $\left(t_{(75)}=1.61, p=0.11\right)$. Following the completion of behavioral testing, rats were anesthetized and perfused, and brain slices were collected as described above. To confirm DREADD expression, floating sections were washed (PBS; $3 \times 10 \mathrm{~min}$ ), blocked ( $0.25 \%$ Triton- $X, 5 \%$ normal goat serum, PBS; $120 \mathrm{~min})$, and incubated with a primary antibody (1:400 rabbit anti-DsRed, $0.25 \%$ Triton-X, $2.5 \%$ normal goat serum, PBS; $24 \mathrm{~h}$; Clontech \#632496, RRID: AB_2737298). Sections were then washed (PBS; $3 \times 10 \mathrm{~min}$ ) and incubated with a secondary antibody conjugated to AF-568 (1:500 goat-anti-rabbit, $0.25 \%$ Triton-X, $2.5 \%$ normal goat serum; PBS, $120 \mathrm{~min}$; Life Technologies \#A11036, RRID: AB_10563566). Finally, sections were washed (PBS; $3 \times 10 \mathrm{~min}$ ), mounted on slides, and coverslipped with Vectashield mounting medium with DAPI. Z-stacks along the rostral/caudal axis of the NAc $(A / P+2.7$ through $A / P+0.5)$ were collected via confocal microscopy to confirm viral expression, quantified as the percent of rats with expression in a particular subregion of the NAc and pseudo-colored to correspond with each chemogenetic experiment. Rats with no detectable DREADD expression in NAc or other areas in examined brain slices (e.g., dorsal striatum, prefrontal cortex) were analyzed as a viral "miss" control group to assess the effects of CNO in rats not expressing DREADDs.

Statistical analysis

Behavioral data were collected using automated procedures (MedAssociates), processed using custom-written Python scripts, and analyzed using Python (V3.6) and GraphPad Prism (V8.0). Addiction severity measures were compared between low- and high-risk rats using unpaired two-tailed $t$-tests. The relative severity distribution was compared between groups using a frequency distribution (range: -10 to +17 , bin size $=1$ ) fit with a nonlinear regression (Gaussian), and the overlap coefficient was calculated between distributions using $R$ (V3.5). The relative contribution of each severity metric to overall severity classification was analyzed using principal component analysis (PCA) in Python: raw behavioral data were normalized, the corresponding covariance matrix and eigens were calculated, and data was replotted on the resulting two-dimensional space. Principal components with an eigenvalue $>1$ were considered significant contributors to overall variance (Tables S1, S2). c-Fos + cell counts along the rostral-caudal axis of the NAc were averaged into a single value per rat, and c-Fos + cells were analyzed using paired two-tailed $t$-tests (eGFP vs DREADD). Lever presses during IntA SA and extinction training (severity $\times$ session) and lever presses during PR and cue-induced reinstatement tests (severity $\times$ treatment) were independently analyzed for each chemogenetic 
a
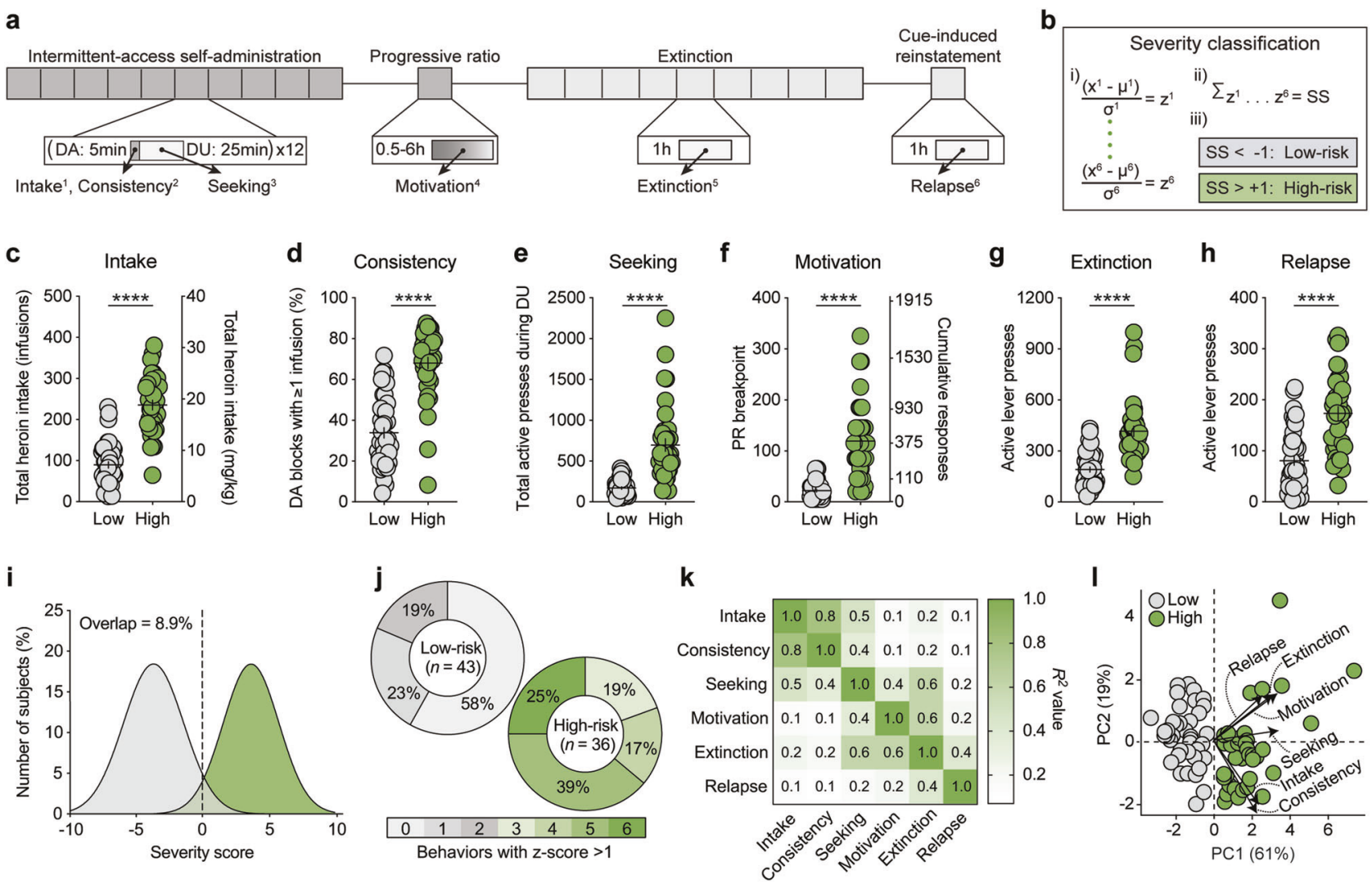

Fig. 1 Heroin self-administration procedure and addiction severity classification. a Timeline of self-administration procedure and behavioral metrics used to classify addiction severity. Dark gray: periods of drug availability; light gray: periods of drug unavailability. (1) Intake: Total heroin intake; (2) Consistency: Percent of drug-available blocks with $\geq 1$ infusion; (3) Seeking: Total active presses during drug-unavailable blocks; (4) Motivation: Breakpoint during PR; (5) Extinction: Total active presses during extinction; (6) Relapse: Total active presses during cue-induced reinstatement. b Severity classification: (i) raw behavioral data were converted to z-scores, (ii) z-scores were combined to give a cumulative severity score (SS), and (iii) individual rats were classified as low-risk (gray) or high-risk (green). c-h Compared to low-risk rats, high-risk rats c self-administered more heroin during IntA SA, d had a higher number of drug-available blocks where they engaged in drug-taking, e pressed the drug-paired lever more during drug-unavailable blocks, $\mathbf{f}$ had higher breakpoints during a PR test, $\mathbf{g}$ pressed the drug-paired lever more during extinction sessions, and $\mathbf{h}$ during the cue-induced reinstatement test. $\mathbf{i}$ Classification produces largely non-overlapping severity distributions between groups. $\mathbf{j}$ High-risk rats scored positive on more severity metrics than low-risk rats. $\mathbf{k}$ Individual severity metrics are all significantly correlated (all $p<0.05$ ), though the relationship between pairs of metrics are highly variable. I Principal component analysis of severity metrics, with individual rats and the original severity metrics (length = eigenvalue, angle = eigenvector) replotted on the resulting two-dimensional space. DA, drug-available; DU, drug-unavailable; IntA, intermittent-access; PC, principal component; PR, progressive ratio; SA, self-administration; ${ }^{* * *} p<0.0001$ (low vs high).

experiment using two-way repeated-measures analysis of variance (RM-ANOVA) followed by Sidak post-hoc tests. The relationship between severity metrics and the effect size of each chemogenetic manipulation on motivation and relapse were independently analyzed using multiple linear regressions. Statistical significance for all analyses was set at $p<0.05$, and data are shown throughout as individual subjects, mean \pm SEM, or best-fit line with $95 \%$ confidence intervals.

\section{RESULTS}

High-risk rats exhibit an addictive phenotype following heroin selfadministration

Six behavioral metrics were normalized to z-scores: (1) intake, (2) consistency, (3) seeking, (4) motivation, (5) extinction, and (6) relapse (Fig. 1a), and combined to give an overall score which revealed addiction-resilient (i.e., low-risk; $n=43$ ) and addiction-vulnerable (i.e., high-risk; $n=36$ ) rats (Fig. $1 \mathrm{~b}$ ). Compared to low-risk rats, high-risk rats self-administered more heroin during IntA training (unpaired $t$-test: $t_{(77)}=10.70, p<0.0001$ ), engaged in more bouts of drug-taking during drug-available blocks (unpaired $t$-test: $t_{(77)}$ $=9.00, p<0.0001)$, had higher levels of drug-seeking during cued drug-unavailable blocks (unpaired $t$-test: $t_{(77)}=7.28, p<0.0001$ ), showed higher motivation to self-administer heroin during $P R$ testing (unpaired $t$-test: $t_{(77)}=8.24, p<0.0001$ ), exhibited higher levels of responding throughout extinction training (unpaired $t$-test: $\left.t_{(77)}=7.03, p<0.0001\right)$, and had greater rates of heroinseeking during cue-induced reinstatement (unpaired $t$-test: $t_{(77)}=$ 5.90, $p<0.0001$; Fig. 1c-h). These behavioral differences resulted in largely non-overlapping addiction severity distributions between low-risk and high-risk rats $(8.9 \%$ overlap; $95 \% \mathrm{Cl}$ : low [ -4.1 to -3.2$]$, high [3.4 to 4.0$]$ ). Nonetheless, there was variability within each subgroup for which severity metrics contributed to their classification and the number of positive severity metrics (low: $0.6 \pm 0.1$; high $4.7 \pm 0.2 ; t_{(77)}=18.92, p<0.0001$; Fig. $1 \mathrm{i}, \mathrm{j}$ ). While the six metrics used for severity classification were all significantly correlated (Fig. S1), the relationship between each metric was highly variable, indicating that the metrics were capturing distinct features of addiction-like behavior (Fig. $1 \mathrm{k}$ ). Dimensional reduction was performed on the severity metrics to identify the vectors accounting for the majority of the variance within the dataset (Table S1). Distribution along the first principal component $(\mathrm{PC} 1)$ corresponded with classification into low-risk $(\mathrm{PC} 1<0)$ or high-risk $(\mathrm{PC} 1>0)$ for all rats (Fig. 1I). PC1 accounted 
a
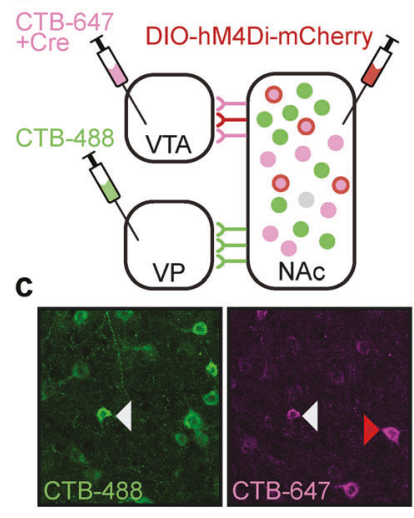

b

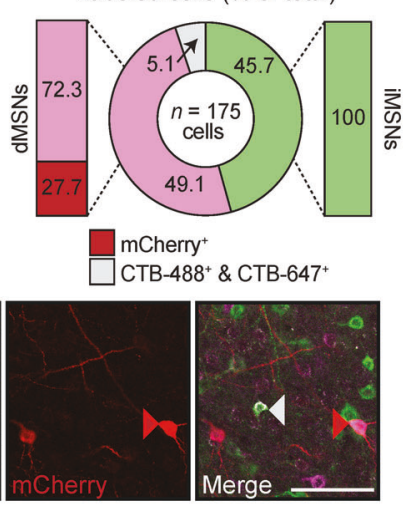

Direct pathway tracing

d

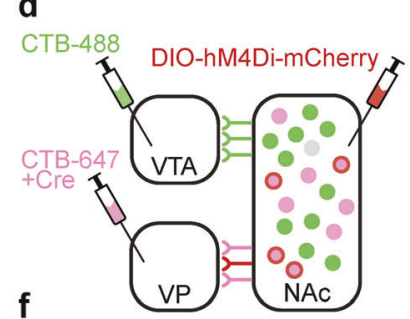

e Labeled cells (\% of total)

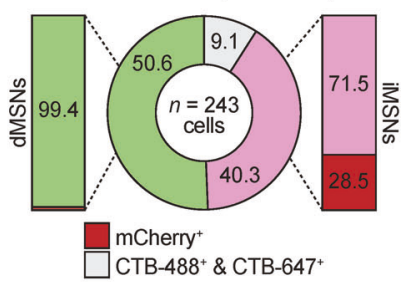

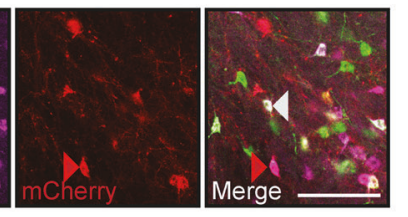

Indirect pathway tracing

Fig. 2 Anatomical tracing of NAc direct and indirect pathways. a Viral strategy for anatomical tracing of NAc dMSNs. b Representative photomicrographs of neuronal labeling and viral expression. c Quantification of neuronal labeling. 30\% of putative dMSNs (magenta) and $0 \%$ of putative iMSNs (green) co-expressed DREADDs (red). d Viral strategy for anatomical tracing of NAc iMSNs. e Representative photomicrographs of neuronal labeling and viral expression. $\mathbf{f}$ Quantification of neuronal labeling. $\sim 30 \%$ of putative iMSNs (magenta) and $<1 \%$ of putative dMSNs (green) co-expressed DREADDs (red). Red arrow: DREADD expressed in cell type of interest; gray arrow: cell co-labeled with CTB-488 and CTB-647; $n=4$ rats, scale bar $=100 \mu \mathrm{m}$.

for $61 \%$ of the cumulative variance and was positively correlated with all severity metrics, while PC2 accounted for $19 \%$ of all variance and was negatively correlated with intake and consistency but positively correlated with all other metrics (Table S2).

\section{Anatomical tracing of NAc direct and indirect pathways}

Reports have indicated that the segregation of MSN output pathways may not be as complete as originally described, with one study in mice finding that $>90 \%$ of dMSNs send collaterals to the VP prior to synapsing in the VTA [27]. Thus, we first sought to determine whether DREADDs could be targeted selectively to MSNs projecting exclusively to the VTA (i.e., dMSNs) or the VP (i.e., iMSNs) in our rats using CTB labeling (Fig. 2a, c, d, f). For the dMSN experiment, we found that 49.1 and $45.7 \%$ of neurons were labeled as putative dMSNs $\left(\right.$ CTB- $\left.647^{+}\right)$and iMSNs $\left(\right.$CTB- $\left.488^{+}\right)$, respectively, while $5.1 \%$ of neurons were co-labeled with both fluorophores. Importantly, $27.7 \%$ of dMSNs were positive for DREADDs, in contrast to $0 \%$ of iMSNs (Fig. 2b). In contrast to what has been reported with mice [27], we did not detect any $\mathrm{DREADD}^{+}$terminals within the $\mathrm{VP}$, suggesting that DREADDtransfected dMSNs were not sending collaterals to the VP in the rats. For the iMSN experiment, we found comparable levels of distinct MSN labeling with $40.3 \%$ and $50.6 \%$ of neurons labeled as putative iMSNs $\left(\mathrm{CTB}-647^{+}\right)$and dMSNs $\left(\mathrm{CTB}-488^{+}\right)$, respectively, and $9.1 \%$ of neurons co-labeled with both fluorophores. DREADD transfection in iMSNs was similar to dMSNs (28.5\%), though a small number of dMSNs $(<1 \%)$ were found to be positive for DREADD expression (Fig. 2e). Given these relative rates, transfection in the small number of dMSNs is unlikely to contribute significantly to alterations in circuit function or behavior.

\section{DREADDs bidirectionally regulate activity of NAc dMSNs and iMSNs}

To confirm that Gi/o- and Gq-DREADDs can modulate activity in NAc dMSNs and iMSNs, we examined the effects of DREADD activation on amphetamine + novelty-induced c-Fos in rats expressing DREADDs in one hemisphere (left NAc) and eGFP in the other (right NAc) (Fig. 3, S2). Inactivation of dMSNs via hM4Di significantly reduced (paired $t$-test: $t_{(3)}=5.00, p=0.0154$ ) while activation of dMSNs via hM3Dq significantly enhanced (paired $t$-test: $t_{(3)}=5.11, p=0.0145$; Fig. 3b, c) the number of NAc c-Fos ${ }^{+}$ cells. Similarly, inactivation of iMSNs via hM4Di significantly reduced (paired $t$-test: $t_{(3)}=3.26, p=0.0473$ ) while activation of
iMSNs via hM3Dq significantly enhanced (paired $t$-test: $t_{(3)}=7.58$, $p=0.0048$; Fig. $3 \mathrm{~d}$, e) the number of c-Fos ${ }^{+}$cells. Together with our tracing data, these findings demonstrate the ability to selectively target and modify the activity of dMSNs or iMSNs with excitatory or inhibitory DREADDs.

dMSN inactivation suppresses reinstatement but not motivation in high-risk rats

To test the hypothesis that an imbalance of signaling between NAc dMSNs and iMSNs drives motivation for heroin selfadministration under a PR schedule of reinforcement and reinstatement of cued heroin-seeking, dual viral-mediated gene transfer was used to selectively express Gi/o- or Gq-DREADDs in each pathway. Given prior work in our lab showing a role for dMSNs in cue-induced reinstatement of cocaine-seeking but not motivation to take cocaine [20], we first sought to determine if transient chemogenetic inhibition of dMSNs had similar effects following heroin self-administration (Fig. 4b). As with the combined group data (Fig. 1), high-risk rats $(n=9)$ exhibited a greater addiction severity profile compared to low-risk rats $(n=9)$, as they self-administered more heroin (severity $\times$ session interaction: $\left.F_{(12,180)}=2.43, p=0.0059\right)$, showed greater motivation for heroin during PR testing (main effect of severity: active presses, $F_{(1,16)}=4.97, p=0.0405$; breakpoint, $\left.F_{(1,16)}=4.18, p=0.0576\right)$, and had higher levels of active lever pressing during extinction training (severity $\times$ session interaction: $F_{(14,210)}=2.80, p=0.0007$ ) and during cue-induced reinstatement (main effect of severity: $F_{(1,16)}=12.92, p=0.0024$; Fig. 4c-f). Both low- and high-risk rats discriminated between active and inactive levers during IntA SA and extinction training (Fig. S3). Notably, increasing Gi/o signaling in NAc dMSNs had no effect on the motivation to self-administer heroin regardless of addiction severity (main effect of treatment: active presses, $F_{(1,16)}=0.28, p=0.60$; breakpoint, $F_{(1,16)}=0.14$, $p=0.71$; linear regression: $r^{2}=0.00, p=0.98$; Fig. 4d) but selectively attenuated reinstatement in high-risk rats (severity $x$ treatment interaction: $F_{(1,15)}=9.86, p=0.0067$ ), with the magnitude of attenuation significantly correlated with an individual's severity score (linear regression: $r^{2}=0.22, p=0.0372$; Fig. 4 f).

dMSN activation exacerbates reinstatement but not motivation in high-risk rats

Given that increasing Gi/o-signaling in dMSNs was sufficient to attenuate reinstatement in high-risk rats, we next tested whether 
TJ O'Neal et al.

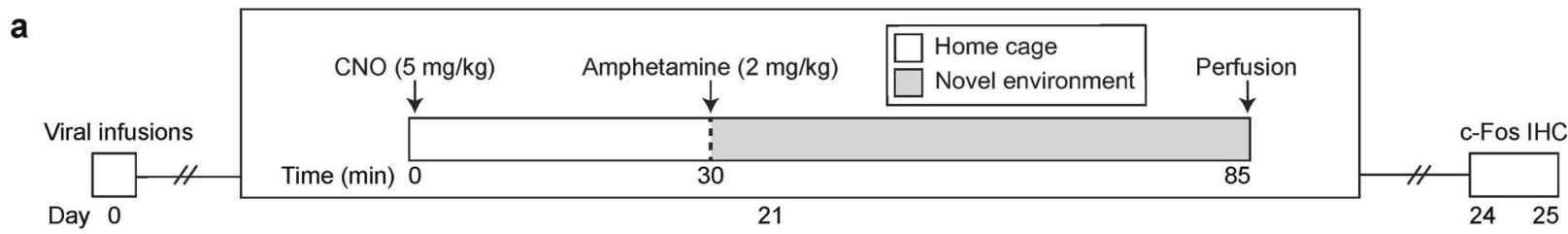

b
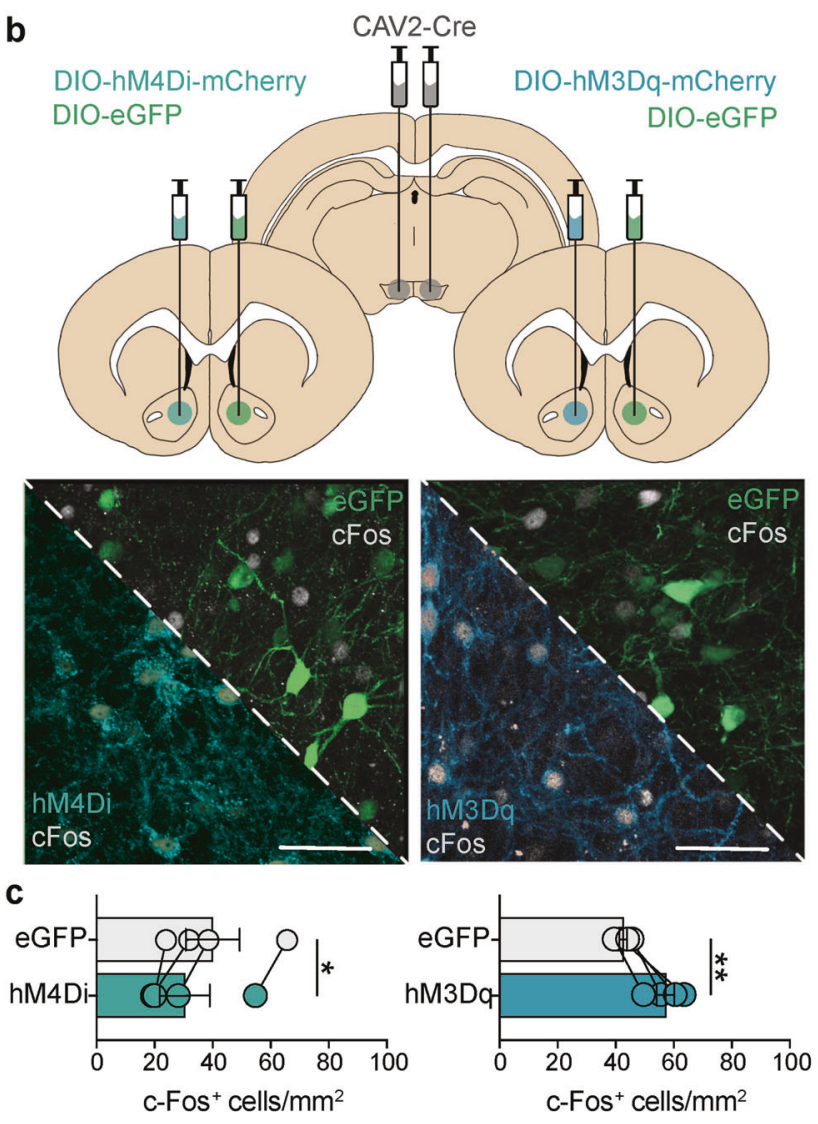
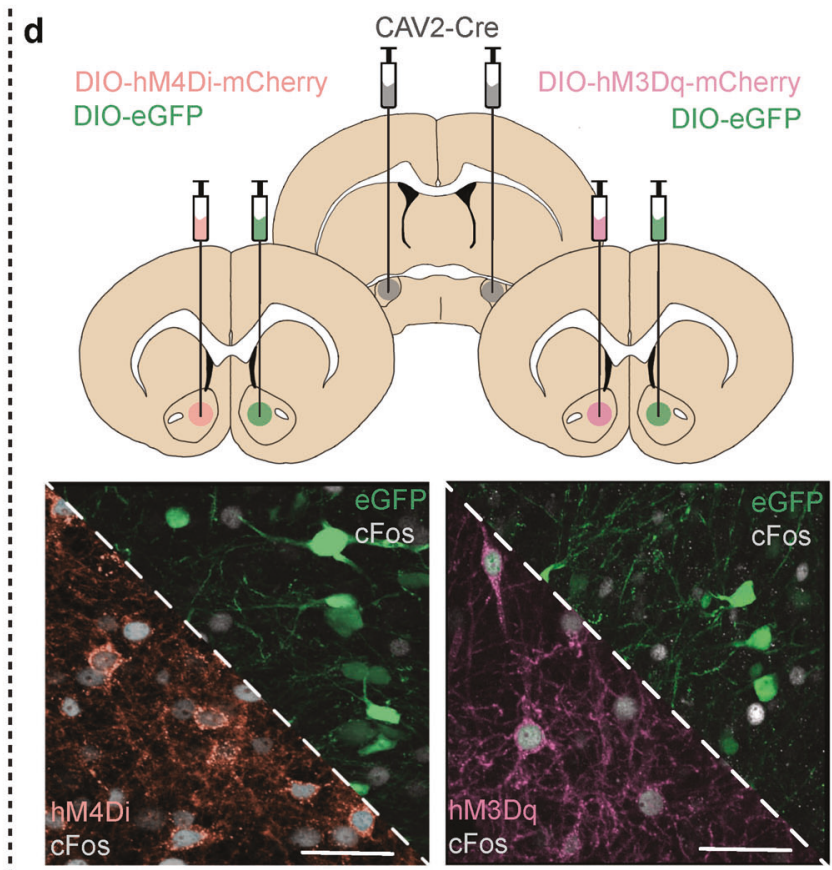

$$
\text { e }
$$
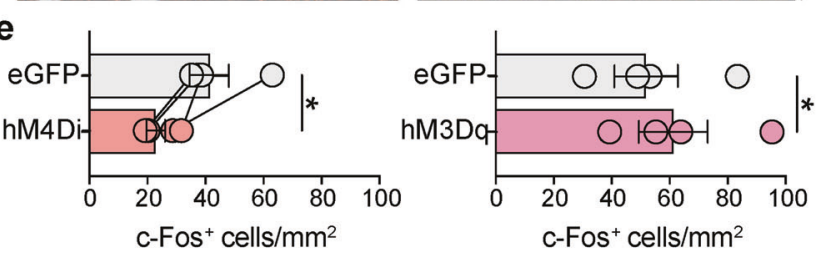

Direct pathway DREADD validation

Fig. 3 DREADDs bidirectionally alter amphetamine-stimulated c-Fos in dMSNs and iMSNs. a Timeline for chemogenetic modulation of amphetamine-stimulated c-Fos activation. b Viral strategy and representative histology for DREADD expression in dMSNs. CAV2-Cre was bilaterally infused into the VTA, DIO-eGFP was unilaterally infused in the right NAc, and DIO-hM4Di or DIO-hM3Dq was unilaterally infused in the left NAc. c Inactivation of dMSNs (via hM4Di) decreases while activation of dMSNs (via hM3Dq) increases the number of c-Fos+ cells in the NAc, relative to eGFP. $\mathbf{d}$ Viral strategy and representative histology for DREADD expression in iMSNs. Infusions were as in (b), except CAV2-Cre was infused into the VP. e Inactivation of iMSNs (via hM4Di) decreases while activation of iMSNs (via hM3Dq) increases the number of c-Fos+ cells in the NAc, relative to eGFP. $n=4$ /group; scale bar $=50 \mu \mathrm{m} ;{ }^{*} p<0.05$ (left NAc vs right NAc), ${ }^{* *} p<0.01$ (left NAc vs right NAc).

transient disruption of the balance between dMSNs and iMSNs by chemogenetic activation of dMSNs could drive reinstatement in low-risk rats (Fig. $4 \mathrm{~g}$ ). As with the other experiments, compared to low-risk rats $(n=11)$, high-risk rats $(n=6)$ self-administered more heroin (severity $\mathrm{x}$ session interaction: $F_{(12,180)}=5.23, p<0.0001$ ), had greater motivation for heroin during PR testing (main effect of severity: active presses, $F_{(1,15)}=38.73, p<0.0001$; breakpoint, $\left.F_{(1,15)}=23.18, p=0.0002\right)$, and had higher responding during extinction sessions (severity $\times$ session interaction: $F_{(14,210)}=3.96$, $p<0.0001)$ and during cue-induced reinstatement of drug-seeking (main effect of severity: $F_{(1,15)}=9.20, p=0.0084$; Fig. 4h-k). Consistent with the inhibition experiment, chemogenetic activation of NAc dMSNs had no effect on the motivation to selfadminister heroin regardless of addiction severity (main effect of treatment: active presses, $F_{(1,15)}=1.50, p=0.24$; breakpoint, $F_{(1,15)}=0.21, p=0.65$; linear regression: $r^{2}=0.0649, p=0.28$; Fig. $4 \mathrm{i})$, but selectively enhanced reinstatement in high-risk rats (severity $\times$ treatment interaction: $F_{(1,15)}=5.41, p=0.0344$ ), exacerbating reinstatement proportionally to individual severity scores (linear regression: $r^{2}=0.2122, p=0.0410$; Fig. 4k).

iMSN activation attenuates reinstatement but not motivation in high-risk rats

Given the well-established role for oppositional control of behavioral output by NAc dMSNs and iMSNs, we next sought to determine whether chemogenetic activation of iMSNs would have similar effects following IntA heroin SA to inactivation of dMSNs (Fig. 5b). Much like what we found with whole group analysis, high-risk rats $(n=8)$ developed a more addictive phenotype than low-risk rats $(n=6)$ (self-administration, severity $x$ session interaction: $F_{(12,144)}=1.97, p=0.0313$; PR active presses, main effect of severity: $F_{(1,12)}=10.98, p=0.0062$; PR breakpoint, main effect of severity: $F_{(1,12)}=11.67, p=$ 0.0051 ; extinction, severity $\times$ session interaction: $F_{(14,168)}=3.02$, 
a

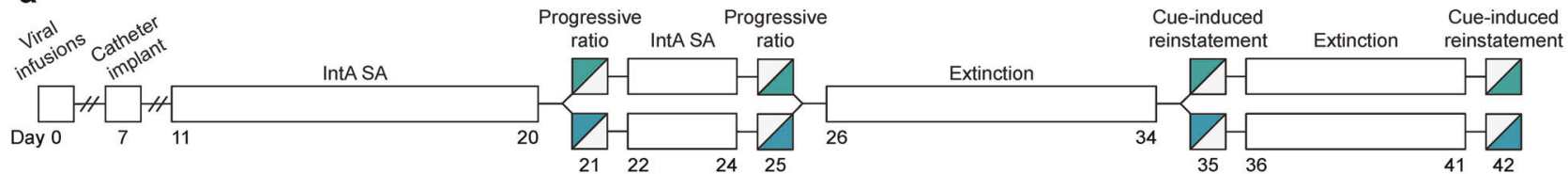

b

C

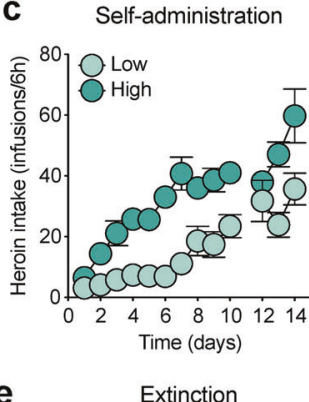

e

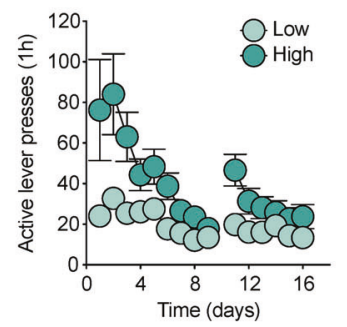

d

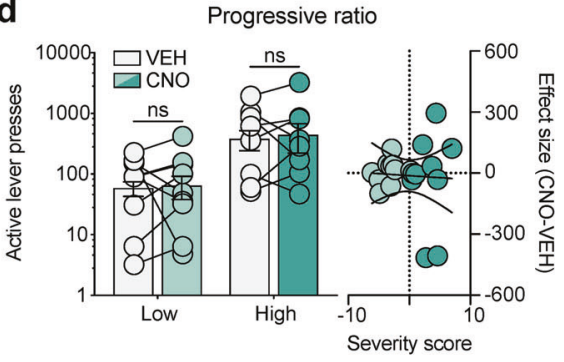

f

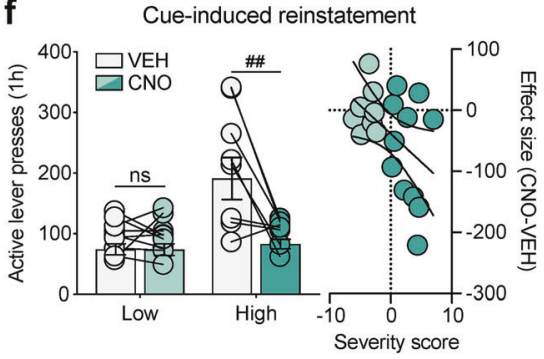

g

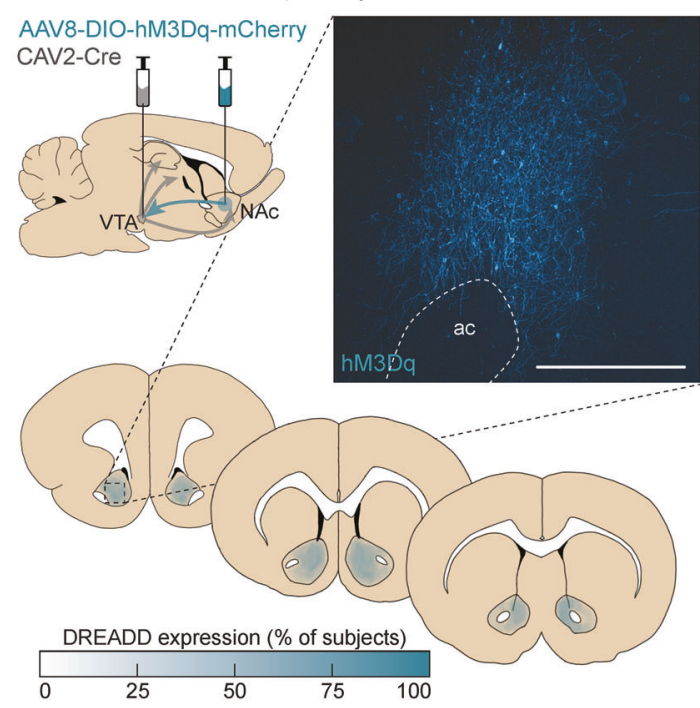

h Self-administration

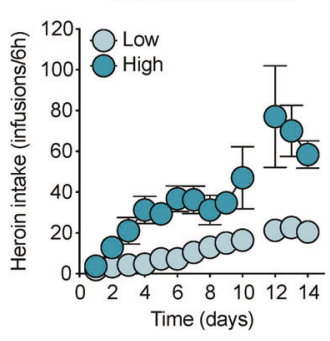

j

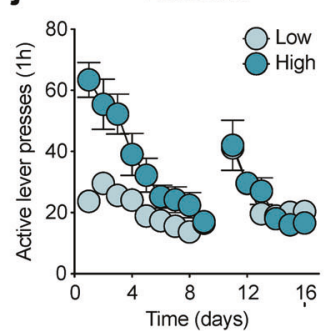

i

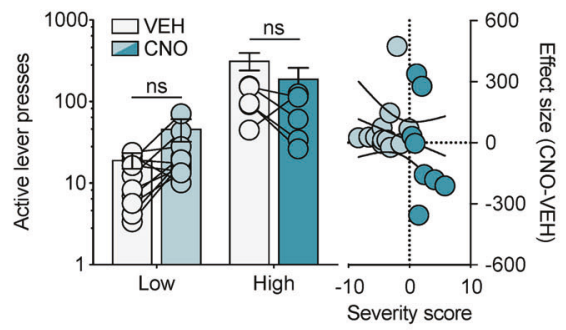

k

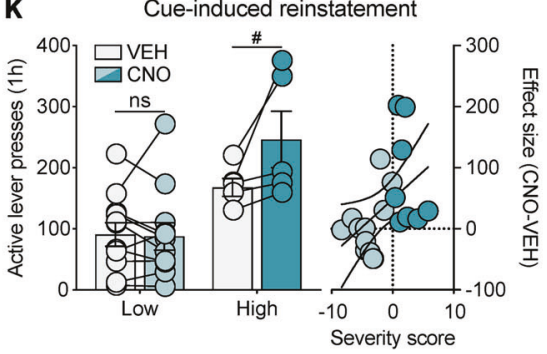

Fig. 4 Transient modulation of dMSNs bidirectionally alters reinstatement but not motivation in high-risk rats. a Timeline for chemogenetic modulation of motivation under a PR schedule of reinforcement and cue-induced reinstatement of drug-seeking. b Viral strategy, representative histology, and quantification of expression for dMSN inactivation. c-f Self-administration, progressive ratio, extinction, and reinstatement data for dMSN-hM4Di rats (low: $n=9$; high: $n=9$ ). d dMSN inactivation has no effect on motivation. Left: High-risk rats have greater responding for heroin than low-risk rats, which is unaffected by dMSN inactivation; right: dMSN-hM4Di has no effect on motivation, regardless of addiction severity. $\mathbf{f} \mathrm{dMSN}$ inactivation selectively reduces reinstatement in high-risk rats. Left: High-risk rats have greater responding for heroin cues than low-risk rats, which is selectively attenuated by CNO; right: the effect size of dMSN-hM4Di significantly correlates with addiction severity. $\mathbf{g}$ Viral strategy, representative histology, and quantification of expression for dMSN activation. $\mathbf{h}-\mathbf{k}$ Selfadministration, progressive ratio, extinction, and reinstatement data for dMSN-hM3Dq rats (low: $n=11$; high: $n=6$ ). i dMSN activation has no effect on motivation. Left: High-risk rats have greater responding for heroin than low-risk rats, which is unaffected by dMSN activation; right: dMSN-hM3Dq has no effect on motivation, regardless of addiction severity. (k) dMSN activation selectively enhances reinstatement in highrisk rats. Left: High-risk rats have greater responding for heroin cues than low-risk rats, which is further enhanced by CNO; right: the effect size of dMSN-hM3Dq significantly correlates with addiction severity. Scale bar $=500 \mu \mathrm{m} ; \mathrm{ac}$, anterior commissure; LV, lateral ventricle; ${ }^{*} p<0.05$ (VEH vs CNO), \# $p<0.01$ (VEH vs CNO). 
a

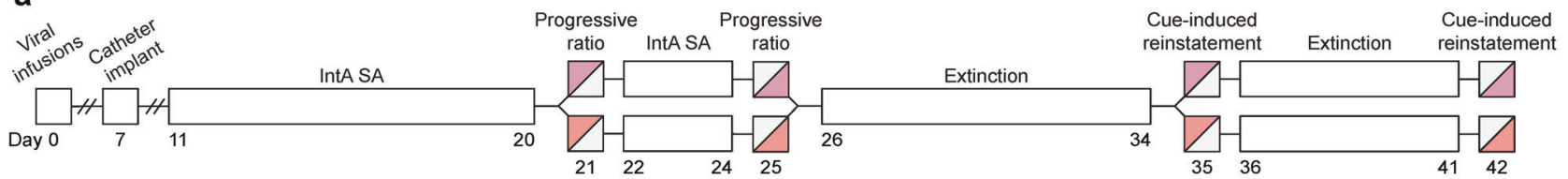

b

Indirect pathway activation

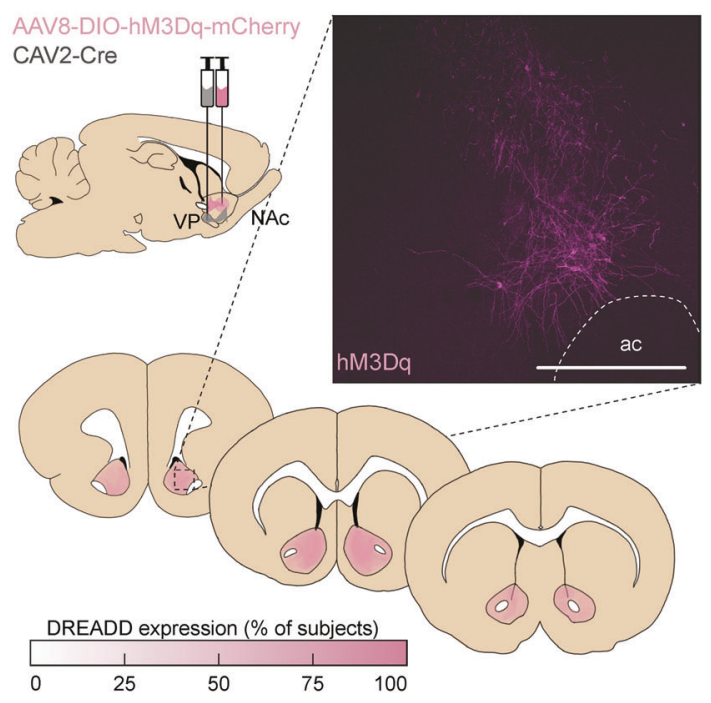

C
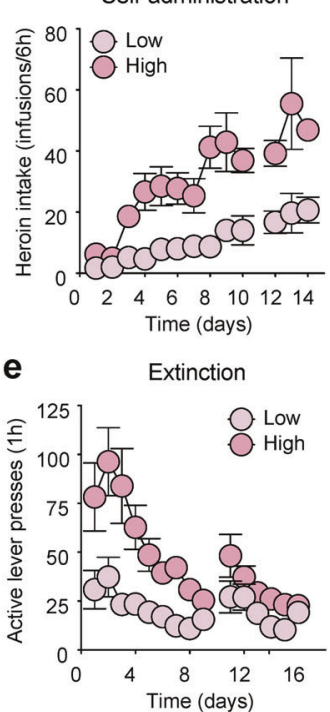

d

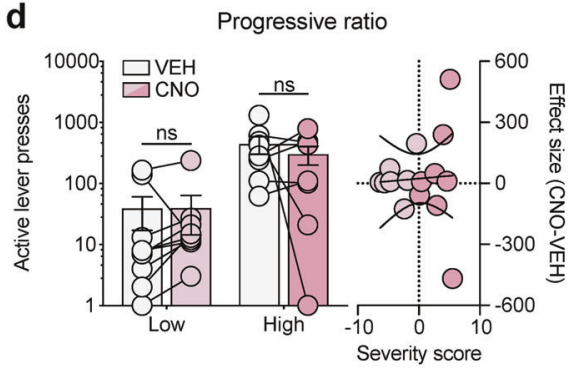

f

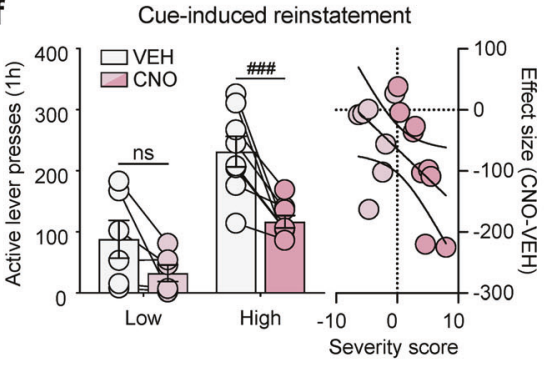

g
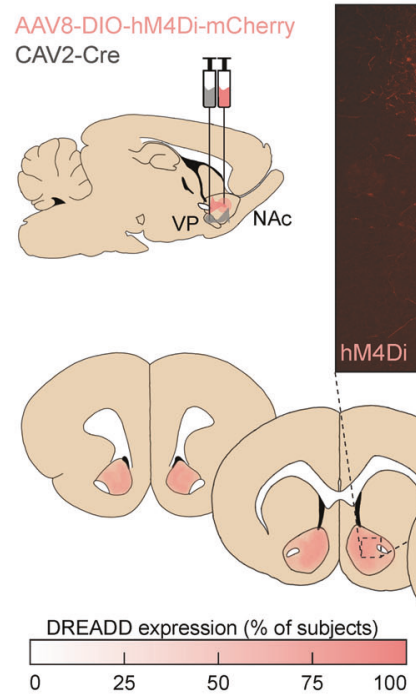

h
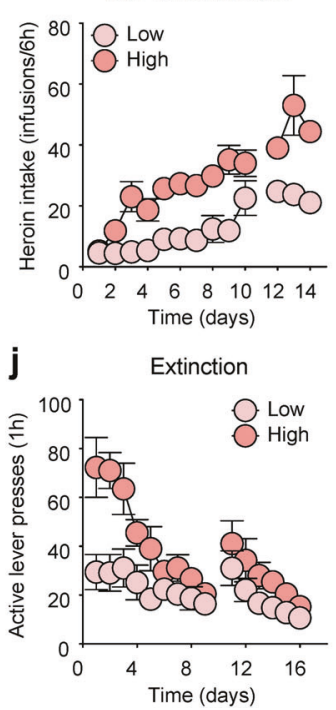

i

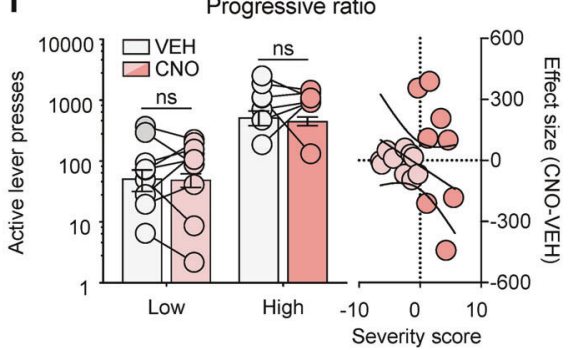

k

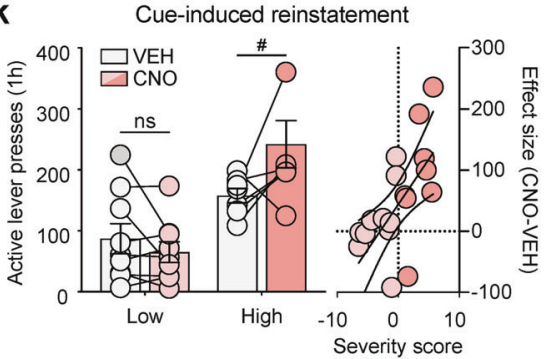

Fig. 5 Transient modulation of iMSNs bidirectionally alters reinstatement but not motivation in high-risk rats. a Timeline for chemogenetic modulation of motivation under a PR schedule of reinforcement and cue-induced reinstatement of drug-seeking. b Viral strategy, representative histology, and quantification of expression for iMSN activation (low: $n=6$; high: $n=8$ ). c-f Self-administration, progressive ratio, extinction, and reinstatement data for iMSN-hM3Dq rats. d iMSN activation has no effect on motivation. Left: High-risk rats have greater responding for heroin than low-risk rats, which is unaffected by iMSN activation; right: CNO has no effect on motivation, regardless of addiction severity. $\mathbf{f}$ iMSN activation selectively reduces reinstatement in high-risk rats. Left: High-risk rats have greater responding for heroin cues than low-risk rats, which is selectively attenuated by CNO; right: the effect size of iMSN-hM3Dq significantly correlates with addiction severity. $\mathbf{g}$ Viral strategy, representative histology, and quantification of expression for iMSN inactivation (low: $n=9$; high: $n=8$ ). $\mathbf{h}-\mathbf{k}$ Selfadministration, progressive ratio, extinction, and reinstatement data for iMSN-hM4Di rats. i iMSN inactivation has no effect on motivation. Left: High-risk rats have greater responding for heroin than low-risk rats, which is unaffected by iMSN inactivation; right: CNO has no effect on motivation, regardless of addiction severity. $\mathbf{k}$ iMSN inactivation selectively enhances reinstatement in high-risk rats. Left: High-risk rats have greater responding for heroin cues than low-risk rats, which is selectively enhanced by CNO; right: the effect size of iMSN-hM4Di significantly correlates with addiction severity. Scale bar $=500 \mu \mathrm{m} ; \mathrm{ac}$, anterior commissure; ${ }^{\#} p<0.05$ (VEH vs CNO), ${ }^{\# \# ~} p<0.001$ (VEH vs CNO). 
$p=0.0004$; cue-induced reinstatement, main effect of severity: $F_{(1,12)}=23.81, p=0.0004$; Fig. 5c-f). Similar to our findings with inhibition of dMSNs, activation of NAc iMSNs had no effect on the motivation to self-administer heroin in high- or low-risk rats (main effect of treatment: active presses, $F_{(1,12)}=0.43, p=0.52$; breakpoint, $F_{(1,12)}=0.54, p=0.48$ ) (linear regression: $r^{2}=0.0027$, $p=0.84$; Fig. $5 \mathrm{~d}$ ); however, the manipulation selectively reduced reinstatement in high-risk rats (severity $\mathrm{x}$ treatment interaction: $\left.F_{(1,12)}=20.15, \quad p=0.0007\right)$, with the degree of reduction significantly correlating with addiction risk (linear regression: $r^{2}=0.2671, p=0.0404 ;$ Fig. 5f). Together, these findings imply a critical role for NAc dMSNs and iMSNs in encoding drug/cue relationships that drive reinstatement of drug-seeking in highrisk individuals.

iMSN inactivation exacerbates reinstatement but not motivation in high-risk rats

To complement these findings, we examined the effect of transient chemogenetic inhibition of iMSNs following heroin self-administration (Fig. $5 \mathrm{~g}$ ). Consistent with our severity classification group data, high-risk rats $(n=8)$ developed a greater addiction-like behavioral profile compared to low-risk rats $(n=9)$ (self-administration, severity $\times$ session interaction: $\left.F_{(12,180}\right)=2.06$, $p=0.0214$; PR active presses, main effect of severity: $F_{(1,15)}=$ 26.57, $p=0.0001$; PR breakpoint, main effect of severity: $F_{(1,15)}=$ 39.86, $p<0.0001$; extinction, severity $\mathrm{x}$ session interaction: $F_{(14,210)}=3.83, p<0.0001$; cue-induced reinstatement, main effect of severity: $F_{(1,15)}=15.48, p=0.0013$; Fig. 5h-k). While increasing Gi/o-signaling in iMSNs had no effect on the motivation to selfadminister heroin in either high- or low-risk rats (main effect of treatment: active presses, $F_{(1,15)}=0.07, p=0.80$; breakpoint, $\left.F_{(1,15}\right)=0.12, p=0.74$; linear regression: $r^{2}=0.0979, p=0.19$; Fig. 5i), it did selectively enhance reinstatement in high-risk rats (severity $\times$ treatment interaction: $F_{(1,15)}=7.62, p=0.0146$ ), with the effect size significantly correlating with individual severity scores (linear regression: $r^{2}=0.383, p=0.0047$; Fig. 5k). Together, these findings suggest that while an imbalance of signaling between dMSNs and iMSNs is likely to contribute to reinstatement of drug-seeking in vulnerable individuals, artificially driving an imbalance is incapable of pushing resilient individuals to reinstate drug-seeking.

\section{CNO administration has no effects in rats lacking DREADD} expression

Recent reports have found that CNO activation of DREADDs occurs indirectly, via retroconversion to clozapine [28]. To ensure that our results were not due to off-target effects, we examined the effects of CNO on motivation and cue-induced reinstatement in rats that had no detectable DREADD expression (viral "miss" rats). This was a heterogeneous group, with similar numbers of direct $(n=6)$ and indirect $(n=7)$ pathway misses (Fig. S5a). Consistent with other groups, high-risk rats $(n=5)$ developed a greater addiction-like behavioral profile compared to low-risk rats $(n=8)$ (self-administration, severity $\mathrm{x}$ session interaction: $F_{(12,132)}=3.11, p=0.0007 ; \mathrm{PR}$ active presses, main effect of severity: $F_{(1,11)}=20.67, p=0.0008$; PR breakpoint, main effect of severity: $F_{(1,11)}=28.15, p=0.0003$; extinction, severity $\mathrm{x}$ session interaction: $F_{(14,154)}=8.95, p<0.0001$; cue-induced reinstatement, main effect of severity: $F_{(1,11)}=10.34, p=$ 0.0082; Fig. S5b-e). Importantly, CNO administration had no effect on motivation (main effect of treatment: active presses, $F_{(1,11)}=0.31, p=0.59$; breakpoint, $F_{(1,11)}=0.65, p=0.44$; linear regression: $\left.r^{2}=0.001, p=0.90\right)$ or cue-induced reinstatement of heroin-seeking (main effect of treatment: $\left.F_{(1,11)}\right)=0.85, p=0.38$; linear regression: $r^{2}=0.01, p=0.73$ ) in high- or low-risk rats (Fig. S5c, e). These results indicate that the observed behavioral effects in the other groups were likely due to the direct actions of CNO on DREADD receptors.

\section{DISCUSSION}

Using a viral DREADD approach, we show that NAc dMSNs and iMSNs have opposing roles in driving heroin-seeking in high-risk rats. These findings lend support to the hypothesis that an imbalance in signaling between these cells underlies addictive behaviors. Specifically, they suggest that heroin use in susceptible individuals likely leads to an increase in direct pathway activity and a concomitant decrease in indirect pathway activity. Nonetheless, given that heroin-seeking could be bidirectionally modulated by both cell types, it is unlikely that changes to direct pathway function were maximal or that there was a complete loss of indirect pathway function in high-risk rats. This is not particularly surprising as iMSNs are generally more excitable and have a greater event frequency under basal conditions than dMSNs [29, 30]. Additionally, we demonstrate that although transient alterations in signaling activity in either pathway are sufficient to both attenuate and exacerbate cue-induced reinstatement of heroin-seeking in high-risk rats, reinstatement behavior was surprisingly unmodifiable in low-risk rats. Of note, chemogenetic modulation of dMSNs and iMSNs had no effect on the motivation to self-administer heroin across phenotype, indicating a divergence in the neural circuits responsible for encoding drugseeking and drug-taking behaviors.

NAc MSNs regulate cue-induced reinstatement of heroin-seeking The NAc plays a critical role in regulating cue-induced reinstatement of seeking across many types of rewards, including heroin [31], cocaine [32-35], methamphetamine [36], alcohol [37], and food $[35,38]$. Moreover, dMSNs and iMSNs have been shown to play oppositional roles in the rewarding and sensitizing effects of cocaine and amphetamine $[23,39,40]$. There has been no work, however, examining the role of these cell types in opioid addiction. Our results show that both cell populations modulate cue-induced reinstatement of heroin-seeking, complementing and expanding previous work showing a similar role for dorsal striatal dMSNs in cued-reinstatement of cocaine-seeking [20]. More surprisingly, though, these results were selective for high-risk rats, suggesting that their role in the encoding of drug cues is specific to conditions that promote the development of addiction-like behaviors. These findings are consistent with previous work demonstrating that synaptic strengthening of NAc iMSNs is enhanced in addiction-resilient mice following cocaine selfadministration [23], and suggest a common role for iMSNs in encoding vulnerability to cue-induced reinstatement of drugseeking. Notably, however, that same study found that chemogenetic inhibition of NAc iMSNs increased the motivation to selfadminister cocaine [23], following previous work showing a role for iMSNs in cocaine-taking [41-43]. Additionally, using a polydrug procedure and behavioral economics, we have recently found that rats have greater motivation for cocaine than heroin [44]. This suggests that psychostimulants and opioids differentially engage motivational circuits, likely due to their unique pharmacological properties (e.g., cocaine increases while morphine decreases cFos activation in iMSNs $[45,46])$. Thus, while dMSNs and iMSNs appear to play a common role in the encoding of drug-seeking, there may be a divergence in the encoding of drug-taking across classes of drugs.

The NAc receives strong glutamatergic input from the prelimbic (PrL) and infralimbic (IL) regions of the medial prefrontal cortex (mPFC), and previous work has found that mPFC, as well as its projections to the NAc, play a critical role in the encoding of cue value and cue-induced reinstatement of drug-seeking [36, 47-49]. Thus, given that modulating activity of subpopulations of NAC MSNs had no effect on reinstatement of drug-seeking in low-risk rats, it is possible that the cue associations formed in MPFC to NAC projections of these rats were insufficiently strengthened during heroin self-administration. The PrL and IL differ in their projection patterns to the NAc, with the PrL preferentially innervating the 
central NAc core and the IL preferentially innervating the surrounding NAC shell. These divergent signaling pathways are generally thought to encode different types of drug-seeking, with the core encoding cue-induced [5, 31, 50, 51] and the shell encoding context-induced $[9,31,52]$ reinstatement. However, cueinduced reinstatement of cocaine $[53,54]$ or ethanol $[55,56]$ induces c-Fos activation in both the core and shell, and inactivation of either core or shell attenuates cue-induced reinstatement of sucrose-seeking [57]. Additionally, blockade of AMPA receptors in the IL [58] or inhibition of CaMKII activity in the shell [59] blocks cue-induced reinstatement of heroin- or morphine-seeking, respectively, indicating a role for the shell in reinstatement of opioid-seeking. Of note, there is extensive local connectivity between MSNs in the NAc [10], so manipulations of either core or shell are likely to alter overall signaling within the NAc. Consistent with these studies, we found no differences between rats with DREADD expression in both subregions compared to those with expression limited to the core or shell, though additional work specifically investigating the role of these subregions in cue-induced reinstatement of heroin-seeking will add clarity to our understanding of how the C-BG circuit encodes heroin cues.

\section{Experimental caveats and limitations}

The phenotyping approach was designed to capture baseline (i.e., genetic) differences in vulnerability, although with any procedure in outbred animals it is difficult to rule out interactions between genetic and environment factors. In addition, there were no significant differences between high- and low-risk rats in active lever presses during early IntA SA (Fig. 1C-e), and there were no significant differences in inactive presses between groups at any point (Fig. S3). Thus, these data suggests that the differences between low- and high-risk rats are likely due to differences in preference for the amount and/or pattern of heroin that is consumed, rather than differences in learning rates. Nonetheless, it is possible that the DREADD effects were selective for rats with high levels of habitual responding (due to more stimulus-response pairings as a result of higher heroin intake).

It is worth noting that $\mathrm{CNO}$ has been shown to activate DREADDs via retro-conversion to clozapine, an atypical antipsychotic with diverse pharmacology [28]. Importantly, while high doses of CNO are capable of having off-target behavioral effects [28,60], the dose of CNO used in these studies was chosen based on previous work in our lab and others showing no behavioral effects in control rats $[20,39,61-70]$. Moreover, we found that the effects of CNO were both severity- (high-risk but not low-risk) and behavior- (relapse but not motivation) dependent. In addition, CNO had no effect on inactive lever pressing (Fig. S4), nor in rats that lacked DREADD expression. Although the sample size of these viral "miss" groups is relatively small, the difference between low- and high-risk rats in behaviors contributing to the severity score was comparable to that from the groups expressing DREADDs. Thus, it is unlikely that our observed effects on reinstatement in high-risk rats are due to non-DREADD mediated effects.

\section{CONCLUSIONS}

In summary, these data support a critical —and opposing - role for the direct and indirect pathways of the NAc in driving cue-induced reinstatement of heroin-seeking in high-risk rats. Interestingly, altering NAC output was unable to change reinstatement in lowrisk rats, suggesting that NAc regulation of drug-seeking may be dependent on the strength of the associations that are formed between drugs and cues. Future work will investigate the role of discrete projections to the NAc (e.g., PrL, IL) in vulnerability to drug-seeking, since these projections are thought to regulate the consolidation of cue/drug relationships during initial drug use $[48,50,71]$.

\section{FUNDING AND DISCLOSURE}

This work was supported by grants from the National Institute on Drug Abuse (F31DA047012 to T.J.O., R01DA036582 to S.M.F., and P30 DA048736 to Dr. Charles Chavkin) and the National Institute of Neurological Disorders (T32NS99578 to Drs. Jane Sullivan and Rachel Wong). The authors declare no competing interests.

\section{ACKNOWLEDGEMENTS}

We thank Dr. John Neumaier and Dr. Michelle Kelly for providing the CAV2-Cre virus for this work, Dr. Scott Ng-Evans for designing MED-PC programs, and Jordyn Richardson and Grayson Baden for assistance with tissue sectioning and immunohistochemistry.

\section{AUTHOR CONTRIBUTIONS}

T.J.O. and S.M.F. conceived experiments, T.J.O., M.N.N. and K.T. performed experiments, T.J.O., M.N.N. and K.T. analyzed data, T.J.O. and S.M.F. wrote the paper and secured funding.

\section{ADDITIONAL INFORMATION}

Supplementary Information accompanies this paper at (https://doi.org/10.1038/ s41386-019-0571-9).

Publisher's note Springer Nature remains neutral with regard to jurisdictional claims in published maps and institutional affiliations.

\section{REFERENCES}

1. Smyth BP, Barry J, Keenan E, Ducray, K. Lapse and relapse following inpatient treatment of opiate dependence. Ir Med J. 2010;103:176-9.

2. Calabresi P, Picconi B, Tozzi A, Ghiglieri, Di Filippo M. Direct and indirect pathways of basal ganglia: a critical reappraisal. Nature Neurosci. 2014. https://doi.org/ $10.1038 / \mathrm{nn} .3743$.

3. Gerfen $C R$, Surmeier DJ. Modulation of striatal projection systems by dopamine. Annu Rev Neurosci. 2011. https://doi.org/10.1146/annurev-neuro-061010-113641.

4. Peters J, De Vries TJ. Glutamate mechanisms underlying opiate memories. Cold Spring Harb Perspect. Med. 2012. https://doi.org/10.1101/cshperspect.a012088.

5. LaLumiere RT, Kalivas PW. Glutamate release in the nucleus accumbens core is necessary for heroin seeking. J Neurosci. 2008. https://doi.org/10.1523/ jneurosci.5129-07.2008.

6. Suto N, Wise RA, Vezina, P. Dorsal as well as ventral striatal lesions affect levels of intravenous cocaine and morphine self-administration in rats. Neurosci Lett. 2011. https://doi.org/10.1016/j.neulet.2011.02.011.

7. Dworkin SI, Guerin GF, Goeders NE, Smith, JE. Kainic acid lesions of the nucleus accumbens selectively attenuate morphine self-administration. Pharmacol Biochem Behav. 1988. https://doi.org/10.1016/0091-3057(88)90292-4.

8. Alderson HL, Parkinson JA, Robbins TW, Everitt BJ. The effects of excitotoxic lesions of the nucleus accumbens core or shell regions on intravenous heroin self-administration in rats. Psychopharmacology. 2001. https://doi.org/10.1007/ s002130000634.

9. Bossert JM, Gray SM, Lu L, Shaham Y. Activation of group II metabotropic glutamate receptors in the nucleus accumbens shell attenuates context-induced relapse to heroin seeking. Neuropsychopharmacology. 2006. https://doi.org/ 10.1038/sj.npp.1300977.

10. Burke DA, Rotstein HG, Alvarez, VA. Striatal local circuitry: a new framework for lateral inhibition. Neuron. 2017. https://doi.org/10.1016/j.neuron.2017.09.019.

11. Kravitz AV, et al. Regulation of parkinsonian motor behaviours by optogenetic control of basal ganglia circuitry. Nature. 2010. https://doi.org/10.1038/ nature09159.

12. Vowles $\mathrm{KE}$, et al. Rates of opioid misuse, abuse, and addiction in chronic pain. Pain. 2015. https://doi.org/10.1097/01.j.pain.0000460357.01998.f1.

13. Deroche-Gamonet V, Belin D, Piazza PV. Evidence for addiction-like behavior in the rat. Science. 2004. https://doi.org/10.1126/science.1099020.

14. Zimmer BA, Oleson EB, Roberts, DCS. The motivation to self-administer is increased after a history of spiking brain levels of cocaine. Neuropsychopharmacology. 2012. https://doi.org/10.1038/npp.2012.37. 
15. Allain F, Minogianis EA, Roberts DCS, Samaha AN. How fast and how often: the pharmacokinetics of drug use are decisive in addiction. Neurosci Biobehav Rev. 2015. https://doi.org/10.1016/j.neubiorev.2015.06.012.

16. American Psychiatric Association. Diagnostic and statistical manual of mental disorders: DSM-5. American Psychiatric Association. DSM. 2014. https://doi.org/ 10.1176/appi.books.9780890425596.744053.

17. Rogan SC, Roth BL. Remote control of neuronal signaling. Pharmacol Rev. 2011. https://doi.org/10.1124/pr.110.003020.

18. Roth BL. DREADDs for Neuroscientists. Neuron. 2016. https://doi.org/10.1016/j. neuron.2016.01.040.

19. Kremer EJ, Boutin S, Chillon M, Danos O. Canine adenovirus vectors: an alternative for adenovirus-mediated gene transfer. J Virol. 2009. https://doi.org/ 10.1128/jvi.74.1.505-512.2000.

20. Yager LM, Garcia AF, Donckels EA, Ferguson SM Chemogenetic inhibition of direct pathway striatal neurons normalizes pathological, cue-induced reinstatement of drug-seeking in rats. Addict Biol. 2019. https://doi.org/10.1111/ adb.12594.

21. Paxinos G, Watson CRR, Emson, PC. AChE-stained horizontal sections of the rat brain in stereotaxic coordinates. J Neurosci Methods. 1980. https://doi.org/ 10.1016/0165-0270(80)90021-7.

22. Gottås A, et al. Levels of heroin and its metabolites in blood and brain extracellular fluid after i.v. heroin administration to freely moving rats. $\mathrm{Br} J$ Pharmacol. 2013. https://doi.org/10.1111/bph.12305.

23. Bock $R$, et al. Strengthening the accumbal indirect pathway promotes resilience to compulsive cocaine use. Nat Neurosci. 2013. https://doi.org/10.1038/nn.3369.

24. Ferguson SM, Norton CS, Watson SJ, Akil H, Robinson, TE. Amphetamine-evoked c-fos mRNA expression in the caudate-putamen: the effects of DA and NMDA receptor antagonists vary as a function of neuronal phenotype and environmental context. J Neurochem. 2003. https://doi.org/10.1046/j.14714159.2003.01815.x.

25. Guettier JM, et al. A chemical-genetic approach to study $G$ protein regulation of $\beta$ cell function in vivo. Proc Natl Acad Sci USA. 2009. https://doi.org/10.1073/ pnas.0906593106.

26. Jain S, et al. Chronic activation of a designer $\mathrm{Gq}$-coupled receptor improves $\beta$ cell function. J Clin Invest. 2013. https://doi.org/10.1172/JCl66432.

27. Pardo-Garcia TR, et al. Ventral pallidum is the primary target for accumbens D1 projections driving cocaine seeking. J Neurosci. 2019. https://doi.org/10.1523/ JNEUROSCI.2822-18.2018.

28. Gomez JL, et al. Chemogenetics revealed: DREADD occupancy and activation via converted clozapine. Science. 2017. https://doi.org/10.1126/science.aan2475.

29. Planert H, Berger TK, Silberberg G. Membrane properties of striatal direct and indirect pathway neurons in mouse and rat slices and their modulation by dopamine. PLoS ONE. 2013. https://doi.org/10.1371/journal.pone.0057054.

30. Calipari ES, et al. In vivo imaging identifies temporal signature of D1 and D2 medium spiny neurons in cocaine reward. Proc Natl Acad Sci USA. 2016. https:// doi.org/10.1073/pnas.1521238113.

31. Bossert JM, Poles GC, Wihbey KA, Koya E, Shaham Y. Differential effects of blockade of dopamine D1-family receptors in nucleus accumbens core or shell on reinstatement of heroin seeking induced by contextual and discrete cues. J Neurosci. 2007. https://doi.org/10.1523/jneurosci.3926-07.2007.

32. Yee J, et al. Muscarinic acetylcholine receptors in the nucleus accumbens core and shell contribute to cocaine priming-induced reinstatement of drug seeking. Eur J Pharmacol. 2011. https://doi.org/10.1016/j.ejphar.2010.10.045.

33. Stefanik MT, et al. Optogenetic inhibition of cocaine seeking in rats. Addict Biol. 2013. https://doi.org/10.1111/j.1369-1600.2012.00479.x.

34. Heinsbroek JA, et al. Loss of plasticity in the D2-accumbens pallidal pathway promotes cocaine seeking. J Neurosci. 2017. https://doi.org/10.1523/ JNEUROSCI.2659-16.2016.

35. Guercio LA, Schmidt HD, Pierce, RC. Deep brain stimulation of the nucleus accumbens shell attenuates cue-induced reinstatement of both cocaine and sucrose seeking in rats. Behav Brain Res. 2015. https://doi.org/10.1016/j. bbr.2014.12.025.

36. Rocha A, Kalivas PW. Role of the prefrontal cortex and nucleus accumbens in reinstating methamphetamine seeking. Eur J Neurosci. (2010). https://doi.org/ 10.1111/j.1460-9568.2010.07134.x.

37. Perry CJ, McNally GP. $\mu$-Opioid receptors in the nucleus accumbens shell mediate context-induced reinstatement (renewal) but not primed reinstatement of extinguished alcohol seeking. Behav Neurosci. 2013. https://doi.org/10.1037/ a0032981.

38. Guy EG, Choi E, Pratt, WE. Nucleus accumbens dopamine and muopioid receptors modulate the reinstatement of food-seeking behavior by food-associated cues. Behav Brain Res. 2011. https://doi.org/10.1016/j. bbr.2011.01.024.
39. Ferguson SM, et al. Transient neuronal inhibition reveals opposing roles of indirect and direct pathways in sensitization. Nat Neurosci. 2011. https://doi.org/ 10.1038/nn.2703.

40. Lobo MK, et al. Cell type-specific loss of BDNF signaling mimics optogenetic control of cocaine reward. Science. 2010. https://doi.org/10.1126/ science.1188472.

41. Barrett AC, Miller JR, Dohrmann JM, Caine, SB. Effects of dopamine indirect agonists and selective D1-like and D2-like agonists and antagonists on cocaine self-administration and food maintained responding in rats. Neuropharmacology. 2004. https://doi.org/10.1016/j.neuropharm.2004.07.007.

42. Caine SB, Negus SS, Mello NK, Bergman J. Effects of dopamine D(1-like) and D(2like) agonists in rats that self-administer cocaine. J Pharmacol Exp Ther. 1999. http://jpet.aspetjournals.org/content/291/1/353.

43. Caine SB, Negus SS, Mello, NK. Effects of dopamine D(1-like) and D(2-like) agonists on cocaine self- administration in rhesus monkeys: Rapid assessment of cocaine dose-effect functions. Psychopharmacology. 2000. https://doi.org/ $10.1007 / \mathrm{s} 002130050023$.

44. Crummy EA, Donckels EA, Baskin BM, Bentzley BS, Ferguson SM. The impact of cocaine and heroin drug history on motivation and cue sensitivity in a rat model of polydrug abuse. Psychopharmacology. 2019. https://doi.org/10.1007/s00213019-05349-2.

45. Uslaner J, et al. Amphetamine and cocaine induce different patterns of c-fos mRNA expression in the striatum and subthalamic nucleus depending on environmental context. Eur J Neurosci. 2001. https://doi.org/10.1046/j.0953816X.2001.01574.x.

46. Ferguson SM, Thomas MJ, Robinson, TE. Morphine-induced c-fos mRNA expression in striatofugal circuits: modulation by dose, environmental context, and drug history. Neuropsychopharmacology. https://doi.org/10.1038/sj. npp.1300465.

47. Rubio FJ, et al. Prelimbic cortex is a common brain area activated during cueinduced reinstatement of cocaine and heroin seeking in a polydrug selfadministration rat model. Eur J Neurosci. 2019. https://doi.org/10.1111/ejn.14203.

48. McGlinchey EM, James MH, Mahler SV, Pantazis C, Aston-Jones G. Prelimbic to accumbens core pathway is recruited in a dopamine-dependent manner to drive cued reinstatement of cocaine seeking. J Neurosci. 2016. https://doi.org/10.1523/ jneurosci.1291-15.2016.

49. Augur IF, Wyckoff AR, Aston-Jones G, Kalivas PW, Peters J. Chemogenetic activation of an extinction neural circuit reduces cue-induced reinstatement of cocaine seeking. J Neurosci. 2016. https://doi.org/10.1523/jneurosci.077316.2016.

50. Reiner DJ, Fredriksson I, Lofaro OM, Bossert JM, Shaham, Y. Relapse to opioid seeking in rat models: behavior, pharmacology and circuits. Neuropsychopharmacology. 2019. https://doi.org/10.1038/s41386-018-0234-2.

51. Rogers JL, Ghee S, See, RE. The neural circuitry underlying reinstatement of heroin-seeking behavior in an animal model of relapse. Neuroscience. 2008 https://doi.org/10.1016/j.neuroscience.2007.10.012.

52. Bossert JM et al. Role of projections from ventral medial prefrontal cortex to nucleus accumbens shell in context-induced reinstatement of heroin seeking. J Neurosci. 2012. https://doi.org/10.1523/JNEUROSCI.0005-12.2012.

53. Kufahl PR, Pentkowski NS, Heintzelman K, Neisewander JL. Cocaine-induced Fos expression is detectable in the frontal cortex and striatum of rats under isoflurane but not a-chloralose anesthesia: Implications for FMRI. J Neurosci. Methods. 2009. https://doi.org/10.1016/j.jneumeth.2009.05.012.

54. Mahler SV, Aston-Jones GS. Fos activation of selective afferents to ventral tegmental area during cue-induced reinstatement of cocaine seeking in rats. J Neurosci. 2012. https://doi.org/10.1523/JNEUROSCI.2277-12.2012.

55. Dayas CV, Liu X, Simms JA, Weiss, F. Distinct patterns of neural activation associated with ethanol seeking: effects of naltrexone. Biol Psychiatry. 2007. https:// doi.org/10.1016/j.biopsych.2006.07.034.

56. Zhao Y, et al. Activation of group II metabotropic glutamate receptors attenuates both stress and cue-induced ethanol-seeking and modulates c-fos expression in the hippocampus and amygdala. J Neurosci. 2006. https://doi.org/10.1523/ JNEUROSCI.2384-06.2006.

57. Lin, P, Pratt, WE Inactivation of the nucleus accumbens core or medial shell attenuates reinstatement of sugar-seeking behavior following sugar priming or exposure to food-associated cues. PLoS ONE. 2014. https://doi.org/10.1371/ journal.pone.0099301.

58. Chen W, et al. Activation of AMPA receptor in the infralimbic cortex facilitates extinction and attenuates the heroin-seeking behavior in rats. Neurosci Lett. 2016. https://doi.org/10.1016/j.neulet.2015.11.024.

59. Liu Z, Zhang JJ, Liu XD, Yu, LC. Inhibition of CaMKII activity in the nucleus accumbens shell blocks the reinstatement of morphine-seeking behavior in rats. Neurosci. Lett. 2012. https://doi.org/10.1016/j.neulet.2012.05.003. 
60. MacLaren $\mathrm{D}$, et al. Clozapine $\mathrm{N}$-oxide administration produces behavioral effects in long-evans rats: implications for designing DREADD experiments. eNeuro. 2016;3:ENEURO.0219-16.2016.

61. Mahler SV, et al. Designer receptors show role for ventral pallidum input to ventral tegmental area in cocaine seeking. Nat Neurosci. 2014. https://doi.org/ 10.1038/nn.3664.

62. Wunsch AM, et al. Chemogenetic inhibition reveals midline thalamic nuclei and thalamo-accumbens projections mediate cocaine-seeking in rats. Eur J Neurosci. 2017. https://doi.org/10.1111/ejn.13631.

63. Wirtshafter D, Stratford TR. Chemogenetic inhibition of cells in the paramedian midbrain tegmentum increases locomotor activity in rats. Brain Res. 2016. https:// doi.org/10.1016/j.brainres.2015.12.014.

64. DiBenedictis BT, Olugbemi AO, Baum MJ, Cherry JA. DREADD-induced silencing of the medial olfactory tubercle disrupts the preference of female mice for oppositesex chemosignals. eNeuro 2015. https://doi.org/10.1523/eneuro.0078-15.2015.

65. Peñagarikano $\mathrm{O}$, et al. Exogenous and evoked oxytocin restores social behavior in the Cntnap2 mouse model of autism. Sci Transl Med. 2015. https://doi.org/ 10.1126/scitranslmed.3010257.
66. Soumier A, Sibille E. Opposing effects of acute versus chronic blockade of frontal cortex somatostatin-positive inhibitory neurons on behavioral emotionality in mice. Neuropsychopharmacology. 2014. https://doi.org/10.1038/ npp.2014.76

67. Zink AN, Bunney PE, Holm AA, Billington CJ, Kotz, CM. Neuromodulation of orexin neurons reduces diet-induced adiposity. Int J Obes. 2018. https://doi.org/10.1038/ ijo.2017.276.

68. Sasaki K, et al. Pharmacogenetic modulation of orexin neurons alters sleep/ wakefulness states in mice. PLoS ONE. 2011. https://doi.org/10.1371/journal. pone.0020360.

69. Garner AR, et al. Generation of a synthetic memory trace. Science. 2012. https:// doi.org/10.1126/science.1214985.

70. Kerstetter KA, et al. Corticostriatal afferents modulate responsiveness to psychostimulant drugs and drug-associated stimuli. Neuropsychopharmacology. 2016. https://doi.org/10.1038/npp.2015.253.

71. Peters J, LaLumiere RT, Kalivas, PW. Infralimbic prefrontal cortex is responsible for inhibiting cocaine seeking in extinguished rats. J Neurosci. 2008. https://doi.org/ 10.1523/jneurosci.1045-08.2008. 